\title{
Topological zero-dimensional photonic modes in chiral coupled-cavity arrays
}

Vassilios Yannopapas

${ }^{\text {*Correspondence: }}$

vyannop@mail.ntua.gr

Department of Physics, National

Technical University of Athens,

Athens, 157 80, Greece

\begin{abstract}
We show theoretically that a properly chosen one-dimensional array of coupled photonic resonators (cavities) may possess localized zero-dimensional topological modes bearing resemblance with the corresponding edge modes/Majorana states in semiconductor nanowires atop a superconducting substrate. These modes constitute a manifold of degenerate states and are robust to the geometrical characteristics of the array, to the dielectric properties of the cavities and of the host medium. Such arrays can be realized in the laboratory as chains of microwave cavities within a metallic wire-network or as lattices of sinusoidally curved dielectric waveguides in the optical regime.
\end{abstract}

PACS Codes: $73.43 .-f ;$ 81.05.Xj; 42.70.Qs

Keywords: chirality; topological states; tight-binding; plasmons; cavities

\section{Introduction}

The topological properties of the quantum states define a new paradigm in the description and classification of condensed matter. Namely, atomic crystals possessing a topologically nontrivial electronic band structure constitute a new class of materials whose salient properties are robust to phase transitions which modify the symmetry order of the atomic solid. Typical examples of such topological solids are the integer/fractional quantum Hall (I/FQHE) systems and the topological insulators. Well-known examples of topological properties are the existence of chiral edge states in QHE systems and the presence of gapless surface states in topological insulators which are both insensitive to the presence of randomness/disorder.

Over the years there has been a continuous transfer of ideas and phenomena from atomic solids to their man-made electromagnetic (EM) counterparts, i.e., photonic crystals and metamaterials. Among several phenomena in traditional condensed matter physics which have found their analogues in artificial electromagnetic systems, the topological properties of matter have been the focus of intensive research in the past years motivated by two main scopes: the investigation of new states of photons in the context of quantum simulation and the design of disorder-immune integrated photonic devices. Examples of topological electronic systems which have been simulated in photonics are 2D lattices of gyromagnetic cylinders simulating the integer QHE [1-12], metamaterials simulating topological insulators [13-18], metamaterial-based microwave networks simulating the fractional QHE [19], and many others.

(c) 2015 Yannopapas; licensee Springer. This is an Open Access article distributed under the terms of the Creative Commons Attribution License (http://creativecommons.org/licenses/by/4.0), which permits unrestricted use, distribution, and reproduction in any medium, provided the original work is properly credited. 
The main application in topological condensed matter comes from the presence of electron states at the boundaries of finite solids, i.e., chiral edge states in 2D systems (e.g., quantum spin Hall effect) and gapless surface states in 3D systems (topological insulators), which are robust to disorder. In finite one-dimensional (1D) systems with non-trivial topology, zero-dimensional localized states may appear in model Hamiltonians such as Kitaev's model [20]. In particular, Kitaev predicted that when a semiconductor nanowire is placed atop a superconducting substrate, Majorana-type fermionic states emerge at the edges of the nanowire; this is a phenomenon with important application in topologically fault-tolerant quantum computing [21-23]. Unfortunately, a proof-of-principle experiment for these zero-dimensional edge states requires a highly sophisticated laboratory setup. Unlike electron systems, topological zero-dimensional states can more easily simulated with photons either in the microwave regime with coupled-cavity arrays [24] or in the optical regime with arrays of metallic nanoparticles [25, 26]. Namely, the EM Green's tensor describing the coupling among the electric dipoles either in EM cavities [24] or nanoparticles [25] as well as the derived photonic frequency bands are in analogy with Bogoliubov-de Gennes equations of Kitaev's model of Majorana edge states in semiconductor nanowires. Besides classical electrodynamics, quantum simulators for Majorana states have been proposed in the context of cold atoms [27] and trapped ions [28].

In the present work we show that an array of coupled cavities immersed in a chiral host medium may support a manifold of zero-dimensional EM modes similarly to the fractional QHE, with obvious application in fault-tolerant quantum computing which is a non-local, decoherence-free type of quantum computing [21-23]. We note that we are working in a regime of high EM-radiation intensity in which case the classical theory of light (Maxwell's equations) is applicable. However, for low intensities where the quantum nature of light (photons) is employed, additional phenomena based on quantum correlations may arise which can possibly enrich the range of applications of the presented system such as, e.g., in multipartite entanglement [29]. The paper is structured as follows. Section 2 presents a tight-binding coupled-dipole formalism which, for suitable EM designs, transforms a classical electrodynamic problem described by Maxwell's equations to an eigenvalue problem equivalent to an electronic tight-binding Hamiltonian of ordinary atomic solids. Section 3 applies the formalism of Section 2 to a coupled-cavity array design which exhibits nontrivial topological frequency bands. Section 4 discusses the emergence of zero-dimensional edges modes in a coupled-cavity array. Section 5 assesses possible experimental realizations of topological coupled-cavity arrays and Section 6 concludes the paper.

\section{Tight-binding coupled-dipole method}

The EM crystals under study here are amenable to a photonic tight-binding description within the framework of the coupled-dipole method [30]. The latter is an exact means of solving Maxwell's equations in the presence of nonmagnetic scatterers. We consider a lattice of cavities within a lossless metallic host. The $i$ th cavity is represented by a dipole of moment $\mathbf{P}_{i}=\left(P_{x ; i}, P_{y ; i}, P_{z ; i}\right)$ which stems from an incident electric field $\mathbf{E}^{i n c}$ and the field which is scattered by all the other cavities of the lattice. This way the polarization vectors of all the cavities are coupled to each other and to the external field leading to the coupleddipole equation

$$
\mathbf{P}_{i}=\alpha_{i}(\omega)\left[\mathbf{E}^{i n c}+\sum_{i^{\prime} \neq i} \mathbf{G}_{i i^{\prime}}(\omega) \mathbf{P}_{i^{\prime}}\right] .
$$


$\mathbf{G}_{i i^{\prime}}(\omega)$ is the electric part of the free-space Green's tensor and $\alpha_{i}(\omega)$ is the polarizability of the $i$ th cavity. Equation (1) is a $3 N \times 3 N$ linear system of equations where $N$ is the number of cavities of the system.

For a particle/cavity of electric permittivity $\epsilon$ embedded within a material host of permittivity $\epsilon_{h}$, the polarizability $\alpha$ is provided by the Clausius-Mossotti formula $\alpha=$ $(3 V / 4 \pi)\left(\epsilon-\epsilon_{h}\right) /\left(\epsilon+2 \epsilon_{h}\right)$, where $V$ is the volume of the particle/cavity. For a lossless Drudetype (metallic) host i.e., $\epsilon_{h}(\omega)=1-\omega_{p}^{2} / \omega^{2}$ (where $\omega_{p}$ is the bulk plasma frequency), the polarizability $\alpha$ exhibits a pole at $\omega_{0}=\omega_{p} \sqrt{2 /(\epsilon+2)}$ (surface plasmon resonance). By making a Laurent expansion of $\alpha$ around $\omega_{0}$ and keeping the leading term [13], we may write $\alpha=F /\left(\omega-\omega_{0}\right) \equiv F / \Omega$ where $F=(27 V / 8 \pi) \omega_{0} \epsilon /(2 \epsilon+4)$. For sufficiently high value of the permittivity of the dielectric cavity the electric field of the surface plasmon is much localized at the surface of the cavity. As a result, in a periodic lattice of cavities, the interaction of neighboring surface plasmons is very weak leading to much narrow frequency bands. By treating such a lattice in a tight binding (TB) manner [13], we may assume that the Green's tensor $\mathbf{G}_{i i^{\prime}}(\omega)$ does not vary much with frequency and therefore, $\mathbf{G}_{i i^{\prime}}(\omega) \simeq \mathbf{G}_{i i^{\prime}}\left(\omega_{0}\right)$. In this case, Eq. (1) becomes an eigenvalue problem

$$
\sum_{i^{\prime} \neq i} \mathbf{G}_{i i^{\prime}}\left(\omega_{0}\right) \mathbf{P}_{i^{\prime}}=\Omega \mathbf{P}_{i}
$$

where $F$ has been absorbed within the definition of $\mathbf{G}_{i i^{\prime}}\left(\omega_{0}\right)$ and we have set $\mathbf{E}^{i n c}=\mathbf{0}$ in Eq. (1) as we seek the eigenmodes of the system of cavities. In the following, we will be dealing with cavities lying in the same plane. We can, therefore, treat separately the case where the electric field lies is perpendicular to the chain axis (transverse modes) from the case where the electric field is parallel to the chain axis (longitudinal modes). In what follows, we will only deal with transverse modes.

In this case, $\mathbf{P}_{i}=\left(P_{x ; i}, P_{y ; i}\right)$ and the Green's tensor $\mathbf{G}_{i i^{\prime}}\left(\omega_{0}\right)$ is given by

$$
\mathbf{G}_{i i^{\prime}}\left(\omega_{0}\right)=F q_{0}^{3} C\left(q_{0}\left|r_{i i^{\prime}}\right|\right) \mathbf{I}_{2}
$$

with $\mathbf{r}_{i i^{\prime}}=\left(x_{i i^{\prime}}, y_{i i^{\prime}}, z_{i i^{\prime}}\right)=\mathbf{r}_{i}-\mathbf{r}_{i^{\prime}}, q_{0}=\omega_{0} \sqrt{\epsilon_{h}\left(\omega_{0}\right)} / c$ and $\mathbf{I}_{2}$ is the $2 \times 2$ unit matrix. The vectors $\mathbf{r}_{i}$ denote the position of the cavities. Since we focus our attention around the surface plasmon frequency $\omega_{0}$, we operate in the subwavelength regime where $q_{0}\left|r_{i i^{\prime}}\right| \ll 1$. In this regime, the function $C\left(q_{0}\left|r_{i i^{\prime}}\right|\right)$ is written as

$$
\begin{aligned}
q_{0}^{3} F C\left(q_{0}\left|r_{i i^{\prime}}\right|\right) & \simeq q_{0}^{3} F \exp \left(\mathrm{i} q_{0}\left|r_{i i^{\prime}}\right|\right) /\left(q_{0}\left|r_{i i^{\prime}}\right|\right) \\
& =t_{i i^{\prime}} \exp \left(\mathrm{i} \phi_{i i^{\prime}}\right),
\end{aligned}
$$

where $t_{i i^{\prime}}$ and $\phi_{i i^{\prime}}$ are real numbers. In what follows, the cavities are connected via coupling elements, i.e., waveguides or transmission lines, in which case the phase factors $\phi_{i j}$ are not necessarily related with the wavevector of the host medium $\epsilon_{h}$ and can therefore be considered as independent parameters.

For a 1D lattice of cavities, we assume the Bloch ansatz for the polarization field, i.e.,

$$
\mathbf{P}_{i}=\left(P_{x ; i}, P_{y ; i}\right)=\mathbf{P}_{n \beta}=\exp \left(\mathrm{i} k R_{n}\right) \mathbf{P}_{0 \beta}
$$


The cavity index $i$ becomes composite, $i \equiv n \beta$, where $n$ enumerates the unit cell and $\beta$ the positions of inequivalent cavities in the unit cell. Also, $R_{n}=n a, n=-\infty \cdots \infty$, denotes the lattice vectors ( $a$ is the lattice period) and $k$ is the Bloch wavevector. By substituting Eq. (5) into Eq. (2) we finally obtain

$$
\sum_{\beta^{\prime}} \tilde{\mathbf{G}}_{\beta \beta^{\prime}}\left(\omega_{0}, k\right) \mathbf{P}_{0 \beta^{\prime}}=\Omega \mathbf{P}_{0 \beta},
$$

where

$$
\tilde{\mathbf{G}}_{\beta \beta^{\prime}}\left(\omega_{0}, k\right)=\sum_{n^{\prime}} \exp \left[\mathrm{i} k\left(R_{n}-R_{n^{\prime}}\right)\right] \mathbf{G}_{n \beta ; n^{\prime} \beta^{\prime}}\left(\omega_{0}\right) .
$$

Solution of Eq. (6) provides the transverse frequency band structure of a 1D periodic system of cavities.

We note that in the Green's tensor of Eq. (3) the Drude metal within which the cavities are formed is an isotropic medium. Since the case of a isotropic chiral host medium [31] will be needed in what follows, we provide the corresponding Green's tensor for the case of a $1 \mathrm{D}$ chain,

$$
\mathbf{G}_{i i^{\prime}}\left(\omega_{0}\right)=F q_{0}^{3} C\left(q_{0}\left|r_{i i^{\prime}}\right|\right) \mathbf{I}_{2}-z_{i i^{\prime}} \mathrm{i} \eta \sigma_{y}
$$

where $\eta$ is the chiral parameter of the host medium and $\sigma_{y}$ is the corresponding Pauli matrix.

\section{Topological frequency bands}

Next we derive the EM Green's tensor for the 1D chain of Figure 1. Namely, we assume that the nearest neighbor (NN) hopping strengths are complex with alternating opposite arguments, $t \exp ( \pm i \phi)$, i.e., the phase factors in the hopping strengths in the chain alternate as, $+\phi,-\phi,+\phi,-\phi,+\phi,-\phi, \ldots$

We note that in EM lattices such as those considered here, a negative phase $-\phi$ can be easily achieved when the cavities are connected e.g., by 1D left-handed transmission lines, i.e. transmission lines supporting backward-propagating waves where the phase velocity is opposite to the group velocity $[32,33]$. Alternatively, the cavities may be connected by waveguides loaded with a left-handed metamaterial. Obviously, a positive phase $+\phi$ can be achieved by similar means (right-handed transmission lines or waveguides loaded with a right-handed material). For the 1D chain of Figure 1, the Green's tensor of Eq. (8) becomes,

$$
\tilde{\mathbf{G}}_{\beta \beta^{\prime}}(k)=\left(\begin{array}{cc}
\mathbf{0}_{2} & \tilde{\mathbf{G}}_{A B} \\
\tilde{\mathbf{G}}_{A B}^{*} & \mathbf{0}_{2}
\end{array}\right),
$$

Figure 1 1D chain of cavities coupled with complex NN hopping strengths. The arrows denote the sign of the phase factor of the (complex) hopping strengths. Due to the dissimilar (opposite) phases in the hopping strengths between a cavity and its left/right first neighbours, the unit cell contains a 'diatomic' basis of length $a$. 
Figure 2 Frequency band structure for $\phi=\pi / 2, I$ $=0.3 a$ of the chain of Figure 1 where the cavities are embedded in a chiral ( $\eta=0.5$ - solid lines) and non-chiral ( $\boldsymbol{\eta}=0$ - dashed lines) host medium. The thick straight line at $\Omega=0$ corresponds to the manifold of degenerate zero-dimensional modes.

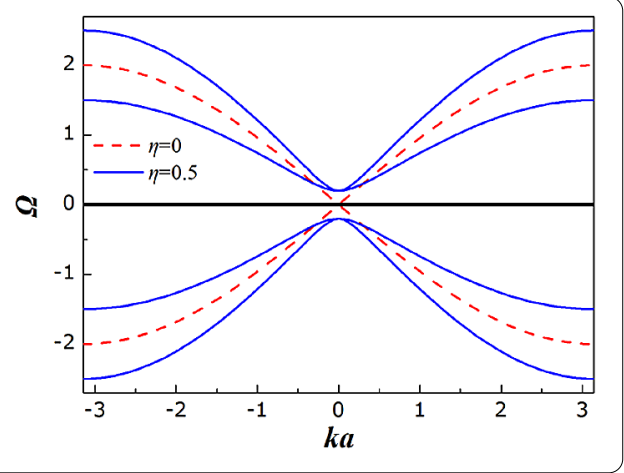

where

$$
\tilde{\mathbf{G}}_{A B}=t\left[\exp (\mathrm{i} \phi)+\exp [-\mathrm{i}(k a+\phi)] \mathbf{I}_{2}-\mathrm{i} \eta[(l-a)+l \exp (-\mathrm{i} k a)] \sigma_{y}\right.
$$

and $\mathbf{0}_{2}$ is the $2 \times 2$ zero matrix.

For a non-chiral medium $(\eta=0)$ the (doubly degenerate) eigenvalues of Eq. (9) (frequency bands) are

$$
\Omega_{ \pm}(k)= \pm 2 t \cos (k a / 2+\phi) .
$$

For $\eta \neq 0$, the expressions for the frequency bands are much more involved and will not be presented here - we only depict them graphically in what follows.

In Figure 2 we show the frequency bands for $\phi=\pi / 2$ and for a nonchiral $(\eta=0)$ and a chiral $(\eta=0.5)$ medium hosting the cavities with $l=0.3 a$. Evidently, a Dirac point $[34,35]$ is present at $k=0$ irrespective of the (nonzero) value of $\phi$. For a non-chiral host medium, the introduction of chirality $(\eta=0.5)$ opens up a frequency gap in place of the Dirac point.

\section{Zero-dimensional topological modes}

The topological nature of the EM modes of the 1D chain of cavities under study can be revealed by assessing the modes of finite chains of Figure 1. The modes of a finite chain of $N$ cavities are found by dropping the Bloch anzsatz of Eq. (5) and by diagonalizing instead the corresponding real-space $4 N \times 4 N$ Green's tensor $\mathbf{G}^{r}$

$$
\mathbf{G}^{r}=\left(\begin{array}{ccccccccc}
G_{i, i} & G_{i, i+1} & 0 & 0 & 0 & \cdots & 0 & 0 & 0 \\
G_{i-1, i} & G_{i, i} & G_{i, i+1} & 0 & 0 & \cdots & 0 & 0 & 0 \\
0 & G_{i-1, i} & G_{i, i} & G_{i, i+1} & 0 & \cdots & 0 & 0 & 0 \\
\vdots & 0 & \ddots & \ddots & \ddots & \cdots & G_{i-1, i} & G_{i, i} & G_{i, i+1} \\
0 & \cdots & \cdots & \cdots & \cdots & \cdots & 0 & G_{i-1, i} & G_{i, i}
\end{array}\right),
$$

where

$$
\mathbf{G}_{i, i}=\left(\begin{array}{cc}
\mathbf{0}_{2} & \mathbf{B} \\
\mathbf{B}^{*} & \mathbf{0}_{2}
\end{array}\right), \quad \mathbf{G}_{i, i+1}=\left(\begin{array}{cc}
\mathbf{0}_{2} & \mathbf{0}_{2} \\
\mathbf{B}^{*} & \mathbf{0}_{2}
\end{array}\right), \quad \mathbf{G}_{i-1, i}=\left(\begin{array}{cc}
\mathbf{0}_{2} & \mathbf{B} \\
\mathbf{0}_{2} & \mathbf{0}_{2}
\end{array}\right)
$$




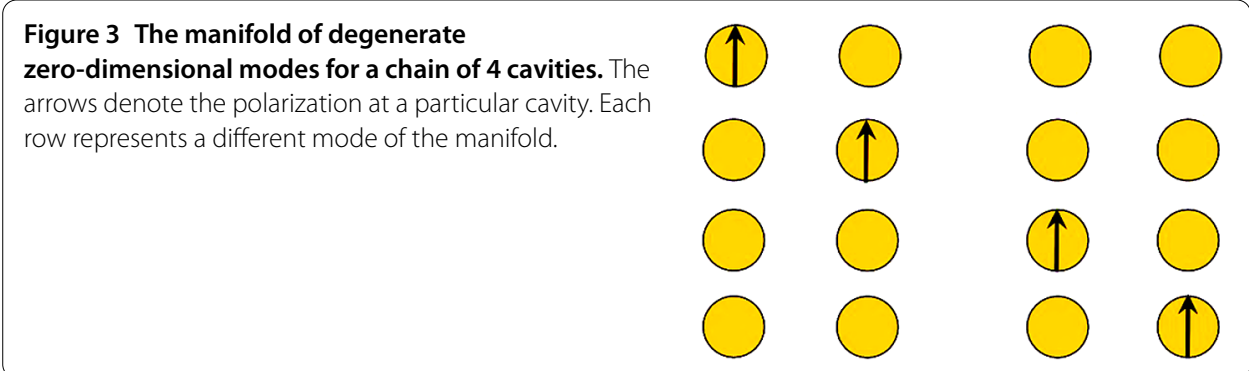

and

$$
\mathbf{B}=t \exp (\mathrm{i} \phi) \mathbf{I}_{2}+\mathrm{i} \eta(a-l) \sigma_{y}
$$

By calculating the eigenspectrum of Eq. (12) we find that half of the eigenmodes, i.e., $2 N$ modes, are ordinary chain modes spread all over the chain whilst the other $2 N$ modes constitute a manifold of modes which are degenerate at $\Omega=0$ (thick straight line at $\Omega=0$ in Figure 2). A similar manifold of states is met in the ground state of a fractional QHE system [36] and in quantum-optics based simulators of the fractional QHE [37]. Each of the degenerate modes is localized at a single cavity as illustrated in Figure 3. Namely, each row of Figure 3 corresponds to a different mode of the chain wherein the polarization vector is zero at all cavities except from one. A similar picture (4 degenerate states) holds when the polarization vectors are normal to the page. All these states constitute a manifold of degenerate states of the chain. The above degenerate zero-dimensional modes may find application in quantum-computing devices with multipartite entangelement [29].

We note that the above zero-dimensional modes are present with $(\eta \neq 0)$ or without the presence of chirality $(\eta=0)$ in the host medium. In the non-chiral case, however, these modes may be fragile since they have a infinitesimal frequency difference from the EM modes spreading over the entire chain (and correspond to the Dirac-cone frequency bands). It is therefore mandatory to generate a frequency band gap at $\Omega=0$ to isolate the degenerate manifold of states from the rest of states. And of course, the band gap should be also topologically nontrivial in order to preserve the degenerate zero-dimensional states. This is achieved by introducing chirality in our system (by assuming that $\eta \neq 0$ in Eqs. (8) and (10)), which is the optical analogue of spin-orbit coupling in electron systems [12]. Due to the non-trivial topological nature of these zero-dimensional EM modes, they are immune to the presence of disorder and/or possible fabrication imperfections [24, 25] facilitating this way their experimental observation. We note that, similarly to the Majorana edge states in Kitaev's model, the observed emergence of zero-dimensional modes in the middle of the frequency gap of Figure 2 signifies the non-trivial topology of the corresponding frequency bands of Figure 2 [26].

\section{Physical realisation}

In order to realize the proposed coupled-cavity arrays in the laboratory, it is important to create a negative phase (argument) of the hopping strength. As stated in Section 2, a negative phase can be achieved in a medium supporting backward-propagating waves, i.e. waves where the phase velocity is opposite to the group velocity. Such media are the socalled negative refractive-index metamaterials or left-handed metamaterials which are artificial dielectrics supporting backward-propagating waves. Therefore, in order to achieve 
Figure 4 Microwave design for a 1D coupled-cavity array with topological properties: cuboid dielectric cavities joined together with metamaterial-based coupling elements and embedded within a 3D network of metallic wires (artificial plasma). The coupling elements are alternately of positive and negative refractive index.

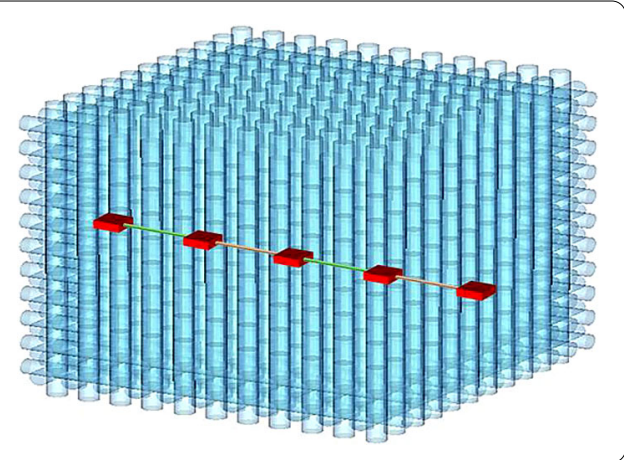

Figure 5 Optical design for a 1D coupled-cavity array with topological properties: a 1D periodic array of sinusoidally curved waveguides.

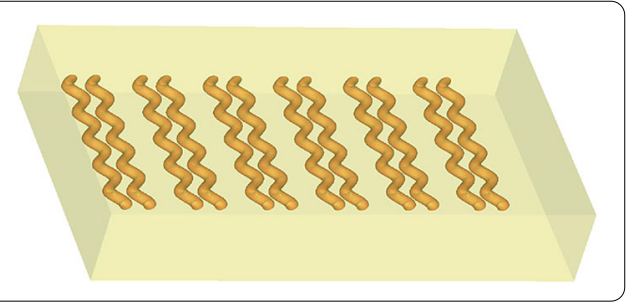

a negative phase coupling, i.e., $\exp (-i \phi)$, between two $\mathrm{NN}$ cavities, the latter must be physically connected via a metamaterial element. Such can be a waveguide loaded with a negative-index metamaterial or a left-handed transmission line [19].

A feasible coupled-cavity array design with desired properties is the one depicted in Figure 4. As our model system requires dielectric cavities within a homogeneous plasma, a lattice of cavities formed within a homogeneous Drude-type metal, e.g., a noble metal $(\mathrm{Au}, \mathrm{Ag}, \mathrm{Cu}$ ), would be the most suitable candidate [38]. However, the plasmon bands are extremely lossy due to the intrinsic absorption of noble metals in the optical regime. Instead, one may use an artificial plasmonic medium operating in the microwave regime where metals are perfect conductors and losses are minimal. Artificial plasma can be created by a 3D network of thin metallic wires of a few tens of $\mu \mathrm{m}$ in diameter and spaced by a few mm [39]. Such a network is background medium hosting the cavity in Figure 4 . The dielectric cavities in Figure 4 are the flat cuboids which are connected via waveguide elements. The waveguide elements are alternately loaded with materials of positive and negative refractive index mimicking the alternation of positive and negative phases. As suggested above, instead of metamaterial-loaded waveguide elements, one can use transmission lines to mimic the phase alteration from cavity to cavity, when operating in the microwave regime [19]. We note that the desired chirality in the host medium can be introduced either by coating the wires with a twisted-nematic liquid crystal with a long pitch or by fabricating a network of metallic helices $[40,41]$ instead of straight wires.

An alternative EM design which can potentially highlight the topological features studied here has been recently realized in the laboratory [42, 43]. Namely, the dispersion relation of Figure 2 with the Dirac singularity (the curve corresponding to $\eta=0$ ) has been reproduced by a 1D periodic array of sinusoidally curved waveguides [44] (see Figure 5) designed so that effective coupling coefficients between adjacent waveguides are alternately positive and negative [42]. The alternative negative and positive coupling is designed by alternating the distances between adjacent waveguides. The sinusoidally curved waveguides are single-mode and are realized by femtosecond direct-writing fabrication [43]. Although 
the topological nature of the corresponding waveguide modes had not been spotlighted in Refs. [42, 43], an experimental verification of the Dirac singularity in the dispersion relation was achieved by means of $1 \mathrm{D}$ conical diffraction [43]. We can therefore hope that a verification of manifold of degenerate zero-dimensional modes reported here is experimentally feasible with the system of Figure 5 .

\section{Conclusion}

We have presented a one-dimensional photonic system, namely an array of coupled cavities in a chiral medium, which is topologically non-trivial and exhibits a manifold of degenerate zero-dimensional modes which are reminiscent of the Majorana states in semiconducting nanowires in touch with a superconducting substrate. This exotic photonic simulator can be realized and probed experimentally system with already fabricated structures such as arrays of sinusoidally curved dielectric waveguides or with cavities coupled with metamaterial elements or transmission lines, embedded in metallic wire networks.

\section{Competing interests}

The author declares that he has no competing interests.

Received: 27 May 2014 Accepted: 14 January 2015 Published online: 01 March 2015

\section{References}

1. Haldane FDM, Raghu S. Possible realization of directional optical waveguides in photonic crystals with broken time-reversal symmetry. Phys Rev Lett. 2008;100:013904.

2. Wang Z, Chong YD, Joannopoulos JD, Soljačić M. Reflection-free one-way edge modes in a gyromagnetic photonic crystal. Phys Rev Lett. 2008;100:013905.

3. Wang Z, Chong YD, Joannopoulos JD, Soljačić M. Observation of unidirectional backscattering-immune topological electromagnetic states. Nature (London). 2009;461:772.

4. Yu Z, Veronis G, Wang Z, Fan S. One-way electromagnetic waveguide formed at the interface between a plasmonic metal under a static magnetic field and a photonic crystal. Phys Rev Lett. 2008;100:023902.

5. Takeda H, John S. Compact optical one-way waveguide isolators for photonic-band-gap microchips. Phys Rev A. 2008;78:023804

6. Han D, Lai Y, Zi J, Zhang ZQ, Chan CT. Dirac spectra and edge states in honeycomb plasmonic lattices. Phys Rev Lett. 2009:102:123904.

7. Ao X, Lin Z, Chan CT. One-way edge mode in a magneto-optical honeycomb photonic crystal. Phys Rev B. 2009:80:033105.

8. Onoda M, Ochiai T. Designing spinning Bloch states in 2D photonic crystals for stirring nanoparticles. Phys Rev Lett. 2009;103:033903.

9. Ochiai T, Onoda M. Photonic analog of graphene model and its extension: Dirac cone, symmetry, and edge states. Phys Rev B. 2009;80:155103.

10. Shen R, Shao LB, Wang B, Xing DY. Single Dirac cone with a flat band touching on line-centered-square optical lattices. Phys Rev B. 2010;81:041410.

11. Poo Y, Wu RX, Lin Z, Yang Y, Chan CT. Experimental realization of self-guiding unidirectional electromagnetic edge states. Phys Rev Lett. 2011;106:093903.

12. Yannopapas V. Photonic analog of a spin-polarized system with Rashba spin-orbit coupling. Phys Rev B. 2011;83:113101.

13. Yannopapas V. Gapless surface states in a lattice of coupled cavities: a photonic analog of topological crystalline insulators. Phys Rev B. 2011:84:195126.

14. Chen WJ, Hang ZH, Dong JW, Xiao X, Wang HZ, Chan CT. Observation of backscattering-immune chiral electromagnetic modes without time reversal breaking. Phys Rev Lett. 2011;107:023901.

15. Zhong W, Zhang X. Dirac-cone photonic surface states in three-dimensional photonic crystal slab. Opt Express. 2011;19:13738

16. Khanikaev AB, Hossein Mousavi S, Tse WK, Kargarian M, MacDonald AH, Shvets G. Photonic topological insulators. Nat Mater. 2012;12:233

17. Liang GQ, Chong YD. Optical resonator analog of a two-dimensional topological insulator. Phys Rev Lett. 2013:110:203904.

18. Rechtsman MC, Zeuner JM, Plotnik Y, Lumer Y, Podolsky D, Dreisow F, Nolte S, Segev M, Szameit A. Photonic Floquet topological insulators. Nature (London). 2013;496:196.

19. Yannopapas $V$. Topological photonic bands in two-dimensional networks of metamaterial elements. New J Phys. 2012;14:113017.

20. Kitaev AY. Unpaired Majorana fermions in quantum wires. Phys Usp. 2001:44:131.

21. Kitaev AY. Fault-tolerant quantum computation by anyons. Ann Phys. 2003;303:2.

22. Alicea J, Oreg Y, von Refael G, Oppen F, Fisher MPA. Non-Abelian statistics and topological quantum information processing in 1D wire networks. Nat Phys. 2011;7:412. 
23. Pachos JK. Introduction to topological quantum computation. Cambridge: Cambridge University Press; 2012.

24. Yannopapas V. Dirac points, topological edge modes and nonreciprocal transmission in one-dimensional metamaterial-based coupled-cavity arrays. Int J Mod Phys B. 2014;28:1441006

25. Poddubny A, Miroshnichenko A, Slobozhanyuk A, Kivshar Y. Topological Majorana states in zigzag chains of plasmonic nanoparticles. ACS Photonics. 2014;1:101.

26. Ling CW, Xiao M, Chan CT, Yu SF, Fung KH. Topological edge plasmon modes between diatomic chains of nanoparticles. arXiv:1401.7520.

27. Bardyn CE, Baranov MA, Rico E, Imamoğlu ZP, Diehl S. Majorana modes in driven-dissipative atomic superfluids with a zero Chern number. Phys Rev Lett. 2012;109:130402.

28. Noh C, Rodríguez-Lara BM, Angelakis DG. Proposal for realization of the Majorana equation in a tabletop experiment. Phys Rev A. 2013;87:040102(R).

29. Liew TCH, Savona V. Multimode entanglement in coupled cavity arrays. New J Phys. 2013;15:025015.

30. Purcell EM, Pennypacker CR. Scattering and absorption of light by non-spherical dielectric grains. Astrophys J. 1973;186:705.

31. Dmitriev V. Space-time reversal symmetry properties of eletromagnetic Green's tensors for complex and bianisotropic media. Prog Electromagn Res. 2004;48:145.

32. Iyer AK, Eleftheriades GV. Negative refraction metamaterials: fundamental principles and applications. New York: Wiley; 2005

33. Caloz C, Itoh T. Electromagnetic metamaterials: transmission line theory and microwave applications. New Jersey: Wiley-IEEE Press; 2006.

34. Sakoda K. Universality of mode symmetries in creating photonic Dirac cones. J Opt Soc Am B. 2012;29:2770.

35. Sakoda K. Proof of the universality of mode symmetries in creating photonic Dirac cones. Opt Express. 2012;20:25181.

36. Laughlin RB. Anomalous quantum Hall effect: an incompressible quantum fluid with fractionally charged excitations. Phys Rev Lett. 1983;50:1395.

37. Cho J, Angelakis DG, Bose S. Fractional quantum Hall state in coupled cavities. Phys Rev Lett. 2008;101:246809.

38. Stefanou N, Modinos A, Yannopapas V. Optical transparency of mesoporous metals. Solid State Commun. 2001;118:69

39. Pendry JB, Holden AJ, Stewart WJ, Youngs I. Extremely low frequency plasmons in metallic mesostructures. Phys Rev Lett. 1996;76:4773.

40. Wu C, Li H, Wei Z, Yu X, Chan CT. Theory and experimental realization of negative refraction in a metallic helix array Phys Rev Lett. 2010;105:247401.

41. Wu C, Li H, Yu X, Li F, Chen C, Chan CT. Metallic helix array as a broadband wave plate. Phys Rev Lett. 2011;107:177401.

42. Efremidis NK, Zhang $P$, Chen Z, Christodoulides DN, Rüter CE, Kip D. Wave propagation in waveguide arrays with alternating positive and negative couplings. Phys Rev A. 2010;81:053817.

43. Zeuner JM, Efremidis NK, Keil R, Dreisow F, Christodoulides DN, Tünnermann A, Nolte S, Szameit A. Optical analogues for massless Dirac particles and conical diffraction in one dimension. Phys Rev Lett. 2012;109:023602.

44. Longhi S, Marangoni M, Lobino M, Ramponi R, Laporta P, Cianci E, Foglietti V. Observation of dynamic localization in periodically curved waveguide arrays. Phys Rev Lett. 2006;96:243901.

\section{Submit your manuscript to a SpringerOpen ${ }^{\circ}$ journal and benefit from:}

- Convenient online submission

Rigorous peer review

- Immediate publication on acceptance

- Open access: articles freely available online

- High visibility within the field

- Retaining the copyright to your article 